\title{
The importance of studying the human intestinal microbiome in its entirety: an interview with Simon Carding
}

\author{
Simon Carding *,1 \\ ${ }^{1}$ Head, Gut Microbes \& Health Research Programme, Quadram Institute Bioscience, Norwich Research Park, Norfolk, NR4 7UA, UK \\ *Author for correspondence: Tel.: +44 0160325 1410; simon.carding@quadram.ac.uk
}
“we must be able to interrogate complexity to understand how it works to keep us healthy and how we can manipulate this for therapy"

First draft submitted: 8 April 2019; Published online: 1 August 2019

Keywords: archaeome $\bullet$ autoimmune disease $\bullet$ bacteriophage $\bullet$ diabetes $\bullet$ inflammatory bowel disease $\bullet$ microbiome $\bullet$ mycobiome $\bullet$ protists $\bullet$ virome

In this exclusive interview, Simon Carding discusses the need for a new perspective in human intestinal microbiome research, with a shift in focus from prokaryotes alone to inclusion of its diversity in its entirety, and how all constituents interact. This interview was conducted by Ellen Colvin, Commissioning Editor of Future Microbiology.

Professor Simon Carding completed postgraduate study at the Medical Research Council Clinical Research Centre (London, UK). He went on to further postdoctoral study at New York University (NY, USA) and at Yale University (CT, USA), prior to beginning his faculty position at the University of Pennsylvania (PA, USA) where he developed a gut biology and immunology research program. Upon returning to the UK, he worked at the University of Leeds (LD, UK) to develop a new program of research focusing on commensal gut bacteria and host-microbe interactions which was developed further upon relocating to the Quadram Institute (Norwich, England) and the University of East Anglia (Norwich, England) to develop a multidisciplinary Gut Biology Research Program. A current focus of his is using commensal gut bacteria-derived microvesicles for the delivery of therapeutic proteins and vaccine antigens to mucosal sites to treat or prevent autoimmune- or infection-related diseases that target the GI-tract and the brain.

\section{Your research is focused on intestinal microbiomes \& how it is much more than just prokaryotes. Can you tell me what you mean by this?}

Microbiome research is technology driven and the technology that is currently available, affordable and accessible is focused on the prokaryote, principally through $16 \mathrm{~S}$ ribosomal RNA-based sequencing. Thus, there has been less attention paid to the other constituents, mainly because studying these is more complicated, less accessible and has fewer tools available. As such looking beyond prokaryotes is difficult, challenging and there is cost to those constraints. However, it has become quite clear that just focusing on bacteria gives an incomplete picture of the microbiome and it is vital to know that, for us to understand how a microbiome functions, its functionality is a product of its complexity and of the interactions between its constituents. Thus, you must study those constituents and their interactions to understand microbiomes in their entirety. This includes the virome, the archaeome, the mycobiome, the protists and includes all kingdoms of life - even plants because they can strongly influence microbiome diversity. This is complex but we must be able to interrogate complexity to understand how it works to keep us healthy and how we can manipulate this for therapy.

\section{How could exploration into other kingdoms of life within the microbiome context contribute to novel disease therapeutics?}

We are already uncovering evidence for the role of elements of the virome in inflammatory bowel disease and diabetes. If you think of the abundance of viruses in the gut that can infect host cells, then you can begin to imagine the enormous potential there is for understanding etiology of a variety of complex inflammatory disorders

Future $\because$ Medicine 
and autoimmune diseases that affect various organs throughout the body including the brain. The virome is of importance, because of its interactions with the prokaryote, and alterations in the prokaryote are or can be correlated with the virome and, particularly, bacteriophages (phages). If phages are lytic and kill bacteria, this could lead to dysbiosis. The problem is, we are currently looking at the microbiome from a one-sided perspective which isolates research. We need to step back and look at the complexity of the system, to include all its constituents. One thing that is often oversimplified, or even ignored, is the contribution of host factors. The microbiome changes and adapts with the host, and as the host changes through ageing, for example, this, too, impacts the microbiome. Thus, we must look at the microbiome in the context of the individual including their lifestyle, environment and behavior.

\section{What do you hope to happen within intestinal microbiome research? What are your predictions for the next 5-10 years? Where will your research target?}

In this field and within my own research, there are mainly observational associations and descriptive studies; these are very detailed and elegant, but we must remember that these are just observations and as such, are not providing much in the way of mechanistic insight. There are still many unanswered questions: How does it all work? What is a 'healthy microbiome' - can it be defined and if so how, and is it the same in everyone? How does the microbiome behave or change the host, for better or for worse? What is the best way of maintaining or restoring a healthy microbiome? Thus, we want to understand the causality and distinguish the effect from the cause. To do this, we need to understand how the microbiome communicates with the host. Already, it is becoming clear that this is unlikely to involve direct interactions between bacterial cells and host cells because of the sterile mucus layer lining of the gut and protecting it from direct contact. This leaves soluble small molecules, produced by microbes, which can cross the mucus barrier. Defining these will then uncover new avenues of, not only just understanding how the microbiome works and interacts, but how we came to be. This will influence bacterial therapy in every way, from small molecule targets to cellular targets - in other words, adding different microbial types, to prebiotics - in other words, adding different substrates for microbes to feed off, all the way through to full microbiome transplantations. Furthermore, this will give a better understanding for which patients require these therapies, and which conditions each is relevant to. It will allow us to tailor therapies to each patient with their own genetic makeup and disease status. At present, it is a bit of a 'shotgun' approach, we treat all with a certain prebiotic or probiotic, or use fecal microbiome transplantation for everyone with one certain disease, but this only works in some cases and not in all. This irregularity is down to a gap in our understanding. With all this in mind, in the next 5-10 years, I would hope that we have a much better insight into how microbes communicate within the gut, as that is the key to developing better therapeutics for individual diseases and patients.

\section{Disclaimer}

The opinions expressed in this interview are those of S Gaisford and do not necessarily reflect the views of Future Medicine Ltd.

\section{Financial \& competing interests disclosure}

The author has no relevant affiliations or financial involvement with any organization or entity with a financial interest in or financial conflict with the subject matter or materials discussed in the manuscript. This includes employment, consultancies, honoraria, stock ownership or options, expert testimony, grants or patents received or pending, or royalties.

No writing assistance was utilized in the production of this manuscript. 